\title{
Endotoxin Modulates the Expression of Renal Drug Transporters in HIV-1 Transgenic Rats
}

\author{
Navaz Karimian Pour and Micheline Piquette-Miller \\ Leslie Dan Faculty of Pharmacy, University of Toronto, Toronto, Ontario, Canada \\ Received, June 11, 2018; Accepted, July 13, 2018; Published, July 14, 2018.
}

\begin{abstract}
PURPUSE: Bacterial co-infections and low grade endotoxemia are common in HIV patients. Inflammation due to endotoxin or HIV may influence the expression and activity of transporters. Kidney transporters influence renal drug clearances including many antiretroviral agents. Our objective was to study the effect of endotoxin and HIV on the renal expression of drug transporters in an HIV-transgenic (HIV-Tg) rat model. These rats develop immune dysfunction and AIDS-associated conditions like humans. METHODS: Endotoxin or saline was administered intraperitoneally to HIV-Tg or wild type (WT) littermates and kidneys were collected 18 hours later. Expression of transporters and cytokines were measured by qRT-PCR and Western blots. Serum cytokine levels were measured by ELISA. RESULTS: Endotoxin induced serum levels of IL-6, TNF- $\alpha$ and IFN$\gamma$ in both HIV-Tg and WT animals. The basal mRNA expression of Oct2, Oct3, Octn1, Mate1, Urat 1 and Ent1was significantly lower (33-60\%) and the expression of Ent2 and Pept2 was significantly higher (33-45\%) in HIV-Tg as compared to WT. While endotoxin significantly downregulated the mRNA expression of Mdra1 and Pept2 in both HIV and WT groups (69-78\%), it imposed a significant reduction on the mRNA expression of Oct2, Oct3, Octn1, Mate1, Oat2, urat1, and Ent1 (54-83\%) only in the WT group. Endotoxin significantly increased the mRNA expression of Pept1 (140\%) in both WT and HIV groups. CONCLUSIONS: HIV and endotoxin each imposed alterations in the expression of many clinically important renal drug transporters although co-infection did not augment this effect. Viral and/or bacterial infections may impact the renal clearance of drug substrates in patients and could potentially be a source of drug-disease interactions.
\end{abstract}

\section{INTRODUCTION}

Infection with HIV virus causes immune dysregulation. This increases the risk of opportunistic infections $(1,2)$. In addition, these patients often suffer from subclinical endotoxemia due to HIV infection-associated disruption of the intestinal mucosal layer which allows microbial translocation of endotoxin from the gut to the systemic circulation $(3,4)$. Endotoxin is a structural element of the outer membrane of Gram-negative bacteria. It stimulates the release of proinflammatory cytokines such as interleukin (IL)-6, IL-1 $\beta$, and tumor necrosis factor (TNF)- $\alpha$ which further contributes to chronic inflammation in patients with HIV (5-8). Numerous studies in animal models have demonstrated endotoxin mediated changes in the expression and activity of drug metabolizing enzymes and drug transporters $(6,7)$. Moreover, the fact that similar changes in drug metabolism are seen in human subjects after administration of endotoxin confirms the ability to translate these preclinical findings to humans (9).
Drug transporters are membrane-bound proteins and based on their mode of action are classified into two super families: 1) ATP binding cassette (ABC) efflux transporters and 2) solute carrier (SLC) uptake transporters (10). Based on their clinical relevance, the International Transporter Consortium has identified several key transporters in the kidney (11). Some are involved in clinical drug-drug interactions such as, the multidrug resistant (MDR)1 protein (also termed P-glycoprotein (P-gp), breast cancer resistance protein (BCRP), organic anion transporter polypeptides (OATPs), organic cation transporter (OCT) 2 and organic anion transporter (OAT) 1 and OAT3 (11). OAT2 has more recently been introduced amongst the emerging transporters of clinical importance. Several transporters are important in efficacy and drug-induced toxicities including multidrug and toxin extrusion transporter

Corresponding Author: Micheline Piquette-Miller, $\mathrm{PhD}$. Professor, Leslie Dan Faculty of Pharmacy, University of Toronto, 144 College Street, Toronto, ON, E-mail: m.piquette.miller@utoronto.ca 


\section{List of Non-Standard Abbreviations:}

ABC, ATP Binding Cassette;

AIDS, Acquired Immunodeficiency Syndrome;

BCRP, Breast Cancer Resistance Protein;

ENT, Equilibrate Nucleotide Transporter;

GAPDH, Glyceraldehyde-3-phosphate

Dehydrogenase;

HIV, Human Immunodeficiency Virus;

HIV-Tg, HIV Transgenic;

IFN- $\gamma$, Interferon Gamma;

IL, Interleukin;

LPS, lipopolysaccharide;

MATE, Multidrug and Toxin Extrusion Transporter;

MDR1, Multidrug Resistant;

MRP, Multidrug Resistance-Associated Protein;

OAT, Organic Anion Transporter;

OATP, Organic Anion Transporter Polypeptide;

OCT, Organic Cation Transporter;

OCTN, Organic Cation/Carnitine Transporter;

PBMC, Peripheral Blood Mononuclear Cells;

PEPT, Peptide Transporters;

P-gp, P-glycoprotein;

qRT-PCR, Real-Time Quantitative Reverse

Transcription Polymerase Chain Reaction;

SLC, Solute Carrier;

Th, T helper;

TNF- $\alpha$, Tumor Necrosis Factor- $\alpha$;

URAT1, Urate Transporter;

WT, Wild type

(MATE1), multidrug resistance-associated proteins (MRPs), equilibrate nucleotide transporters (ENTs), and peptide transporters (PEPTs). Organic cation/carnitine transporters (OCTNTs) and organic anion urate transporter (URAT1) are also important renal transporters involved in the transport of endogenous compounds.

It is well recognized that inflammation can impact the expression and activity of drug transporters in the liver, intestine and blood-brain barrier (6-8). Preclinical studies in HIV transgenic rats and in viral protein treated cells have demonstrated that HIV viral proteins can alter the expression of a number of $\mathrm{ABC}$ transporters (12-15). These findings are supported by recent clinical studies that demonstrated significant decreases in the protein expression of P-gp and MRP2 in the rectosigmoid colon and MRP2 and BCRP in the intestine of HIV-infected patients as compared to healthy individuals $(16,17)$. Much less is known about the impact of inflammation on renal transporters. Many antiviral agents that are used by HIV patients are cleared by renal drug transporters (Table 1) (18). Thus, studying whether their expression is modulated during disease is of clinical relevance. Our primary objective was to investigate the effect of HIV and/ or bacterial co-infection on the expression of renal drug transporters. As both HIV and bacterial infections can impose changes to transporters, we hypothesized that immunologic alterations associated with HIV infection would further impact endotoxin mediated effects. We used a commercially available non-infectious HIV-Tg rat model developed by Reid et al. in 2001 (19). The transgene is comprised of an HIV-1 provirus lacking functional gag and pol genes which are responsible for viral replication. The expression of HIV viral proteins is seen in many tissues including kidneys $(20,21)$. These animals demonstrate immune dysregulation with abnormal $\mathrm{T}$ helper (Th)-1 response but retain normal Th2 function and show clinical manifestations of AIDS by 5-9 months of age (19). Thus, this model provides us with an established method of studying the impact of HIV co-infection on kidney transporters.

The presented study examined the effect of HIV and bacterial co-infection on the expression of renal drug transporters in a HIV transgenic rat model. Our results demonstrated that both HIV disease and bacterial endotoxin altered the expression of clinically important renal drug transporters some of which are responsible for the clearance of antiviral agents. Our work presents novel results, and to our knowledge, this study is the first to show that HIV viral proteins cause changes in the renal expression of SLC drug transporters in a HIV-Tg rat model. It also is the first study that demonstrates the endotoxin mediated effects on some of the SLC drug transporters such as Mate1 in the kidney. This study can provide new insight to potential drug-disease interactions and may help to shed light on the inter and intra-subject variability that is observed in renal clearance of renally excreted drugs.

\section{METHODS}

Animals and experimental design. Hsd-HIV-1 (F344) transgenic male and wild type F344/NHsd, originally purchased from Harlan Laboratories (Indianapolis, IN, USA), were bred in house. HIV$\mathrm{Tg}$ and WT littermates were separated after weaning. Rats were housed in a temperature-controlled facility on a 12-hour light/dark cycle and given free access to water and standard chow. 
Table 1: Antiretroviral substrates of renal drug transporters (adapted from reference (18)).

\begin{tabular}{|c|c|}
\hline $\begin{array}{l}\text { Renal Drug } \\
\text { Transporter }\end{array}$ & Antiretroviral Drug Substrates \\
\hline $\mathrm{P}-\mathrm{gp}^{a}$ & $\begin{array}{l}\text { Abacavir, Atazanavir, Darunavir, Dasabuvir, Didanosine, Emtricitabine, Indinavir, Lamivudine, } \\
\text { Lopinavir, Maraviroc, Nelfinavir, Raltegravir, Ritonavir, Saquinavir, Stavudine, Tenofovir } \\
\text { disoproxil fumarate, Zidovudine }\end{array}$ \\
\hline $\mathrm{MRP}^{b}$ & Darunavir, Indinavir, Lopinavir, Ritonavir, Saquinavir, Tenofovir disoproxil fumarate \\
\hline MRP4 & Abacavir, Nelfinavir, Tenofovir disoproxil fumarate, Zidovudine \\
\hline $\mathrm{BCRP}^{c}$ & Abacavir, Dasabuvir, Dolutegravir, Efavirenz, Lamivudine, Raltegravir, Zidovudine \\
\hline $\mathrm{OCT} 2^{d}$ & Abacavir, Emtricitabine, Lamivudine, Tenofovir disoproxil fumarate, Zalcitabine, Zidovudine \\
\hline MATE1 ${ }^{e}$ & Lamivudine, Tenofovir disoproxil fumarate \\
\hline MATE2, MATE2K & Lamivudine \\
\hline OAT1 ${ }^{f}$ & Didanosine, Lamivudine, Stavudine, Tenofovir disoproxil fumarate, Zalcitabine, Zidovudine \\
\hline OAT3 & Abacavir, Didanosine, Stavudine, Tenofovir disoproxil fumarate, Zalcitabine, Zidovudine \\
\hline OAT4 & Zidovudine \\
\hline OATP1A2 ${ }^{g}$ & Darunavir, Lopinavir, Saquinavir \\
\hline
\end{tabular}

Five-month-old male HIV-Tg rats and their WT litter mates were administered an i.p. injection of either saline (control) or $5 \mathrm{mg} \mathrm{kg}$-1 of bacterial endotoxin (lipopolysaccharide (LPS)) (Escherichia coli 055: B5) (Sigma-Aldrich, Oakville, ON, Canada) (7 per group). This dose was chosen to model acute bacterial infections in which a full immune response is triggered. Animals were anesthetized using isoflurane (Fresenius Kabi Canada, Toronto, ON, Canada) and euthanized 18 hours after injection. Serum and tissues were collected, snap-frozen in liquid nitrogen and stored at $-80^{\circ} \mathrm{C}$ for future analysis. The time point was chosen based on previous studies that demonstrated endotoxin-mediated changes in the expression of drug transporters in other organs (22). A pilot study run in 3-month HIV-Tg and WT rats found relatively similar endotoxin mediated changes in male and female rats (Supplementary Figure 1). We calculated that 6 animals/group were needed to obtain statistically significant differences at the $95 \%$ confidence level based on variability and effect size seen in the pilot study. All animal studies were approved by the Office of Research Ethics at the University of Toronto and conducted in accordance with the guidelines of the Canadian Council on Animal Care.

Serum analysis. Serum concentrations of IFN- $\gamma$, TNF- $\alpha$, and IL- 6 were determined using commercially available enzyme-linked immunosorbent assay (ELISA) kits (R\&D Systems, Minneapolis, MN, USA) according to the manufacturer's instructions. The minimum detectable levels were $12.5,62.5$, and $31.2 \mathrm{pg} / \mathrm{ml}$ for TNF- $\alpha$, IL- 6 , and IFN- $\gamma$, respectively.

Quantitative real time polymerase chain reaction. Total RNA was isolated from $50 \mathrm{mg}$ frozen renal tissue using the TRIZOL (Invitrogen, Carlsbad, CA, USA) extraction method according to the manufacturer's instruction. Purity and concentration of the RNA was measured using a NanoDrop 1000 spectrophotometer (Thermo Fisher Scientific, Waltham, MA, USA). Total RNA samples $(2 \mu \mathrm{g})$ were treated with DNase (Invitrogen) and reversed transcribed to cDNA using a High Capacity cDNA Reverse Transcription Kit (Applied Biosystems, Foster City, CA, USA). The qRT-PCR reaction was performed in triplicates using a Power SYBR Green detection system (ABI HT 7900; Applied Biosystems, Streetsville, ON, Canada) with primers specific for each gene (Supplemental Table 1). A comparative threshold cycle method $(X X \mathrm{CT})$ was used to calculate the relative mRNA expression of each gene of interest normalized to the housekeeping gene glyceraldehyde-3-phosphate dehydrogenase (GAPDH). Similar results were obtained when either GAPDH or $\beta$-actin were used as the housekeeping gene. 

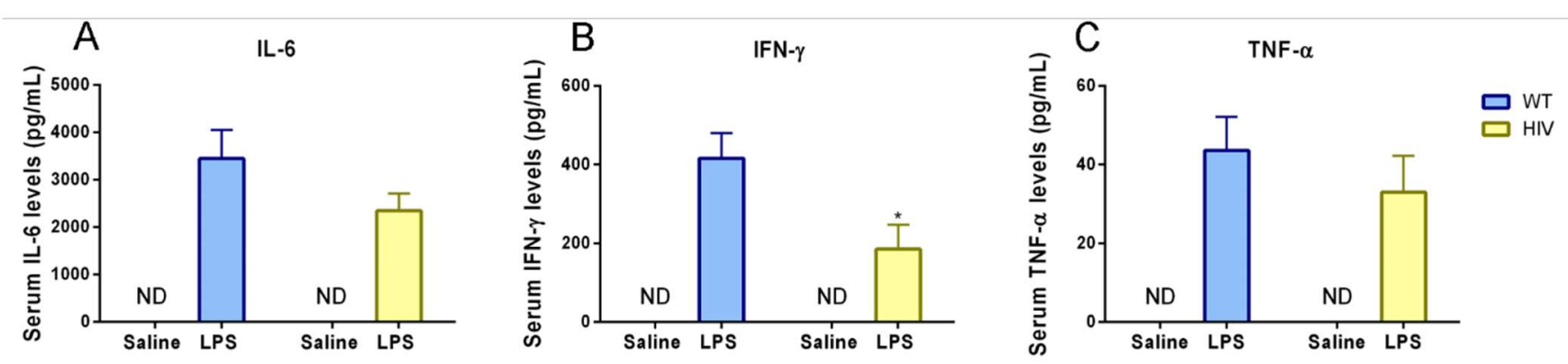

Figure 1. Effect of endotoxin on serum pro-inflammatory cytokines. Serum levels of (A) IL-6, (B) IFN- $\gamma$, and (C) TNF$\alpha$ in WT and HIV-Tg rats $18 \mathrm{~h}$ after i.p. administration of either $5 \mathrm{mg} \mathrm{kg}^{-1}$ LPS or saline ( $\mathrm{n}=7 /$ group). Concentrations were quantified by ELISA as described in materials and methods. Data represents the mean \pm S.E.M. Statistical analysis was performed using Student's t-test. * Significantly different from endotoxin-treated WT $(* \mathrm{p}<0.05)$. ND: not detected, cytokine levels were below the limits of detection.

Western blot analysis. Membrane proteins were extracted from tissues as previously described (23). Briefly, kidney samples (300 mg) were homogenized in lysis buffer (0.1 M Tris-HCL (Sigma-Aldrich), $\mathrm{pH}$ 7.5, $3 \mu \mathrm{L} / \mathrm{ml}$ protease inhibitors cocktail (SigmaAldrich), $50 \mu \mathrm{g} / \mathrm{mL}$ phenylmethylsulfonyl fluoride (PMSF) (Bioshop Canada Inc., Burlington, ON, Canada) and centrifuged at $2500 \mathrm{rpm}$ for $15 \mathrm{~min}$ at $4^{\circ} \mathrm{C}$ (GH-3.8 swing bucket rotor) (Beckman Coulter, California, USA). The supernatant was collected and centrifuged at $34100 \mathrm{rpm}$ for $1 \mathrm{hr}$ at $4^{\circ} \mathrm{C}$ (90 Ti rotor) (Beckman). Protein concentrations were measured using the Bradford assay (Bio-Rad Laboratories, Mississauga, ON, Canada). Samples $(50 \mu \mathrm{g})$ were separated using sodium dodecyl sulfate (SDS)PAGE and transferred to polyvinylidene fluoride (PVDF) membranes (Bio-Rad Laboratories). The membranes were blocked with $5 \%$ milk powder in tris buffered saline (TBST) and incubated with the following primary antibodies overnight: P-gp antibody (1:100), (Cat\# ALX-801-002-C100, Enzo Life Sciences, Farmingdale, NY, USA), PEPT1 (1:100) (Cat\# sc-373742, Santa Cruz Biotechnology, Inc., Dallas, TX, USA), OCT2 (1:1000) (Cat\# sc365116, Santa Cruz Biotechnology, Inc.), OCT3 (1:1000) (Cat\# BS3359, Bioworld Technology, Inc., Louis Park, MN, USA), ENT1 (1:100) (Cat\# sc377283, Santa Cruz Biotechnology, Inc.), MATE1 (1:200) (Cat\# sc-138983, Santa Cruz Biotechnology, Inc.), OAT2 (1:1000) (LS-C313517, LifeSpan BioSciences, Inc., Seattle, WA, USA), UEAT1 (1:1000) (Cat\# URAT11-A, Alpha Diagnostic International, San Antonio, TX, USA. Following several sequential washes, membranes were treated with secondary anti-mouse antibody (1:30000) (Cat\#
NA931, Jackson ImmunoResearch Laboratories, West Grove, PA, USA) in the case of P-GP, PEPT1, OCT2, ENT1, MATE1, or secondary anti-rabbit antibody (1:1000) (Cat\# NA934, GE Healthcare, Mississauga, ON, Canada) in the case of OCT3, OAT2, URAT1. Beta-actin (1:75000) (Cat\# A1978, Sigma-Aldrich, Oakville, ON, Canada) was used as an internal loading control and a calibrator sample was loaded on all gels to control for variability between gels. SuperSignal West Femto Maximum Sensitivity Substrate (ThermoScentific, Rockford, IL, USA) was used for immunodetection and band intensity was determined using Alpha Ease FC imaging software (Alpha Innotech, Santa Clare, AC, USA).

\section{DATA AND STATISTICAL ANALYSIS}

Prism 6 software (GraphPad Software Inc., La Jolla, CA, www.graphpad.com) was used for statistical analysis. ANOVA was used to determine the statistical differences among the groups. Post hoc multiple comparisons were performed using the Bonferroni multiple comparison tests. All data are presented as the mean \pm SEM.

\section{RESULTS}

HIV-Tg rat characteristics. At 5 months of age, the mean body weight of HIV-Tg animals was $20 \%$ lower than that of their WT littermates (HIV-Tg: 336 $\pm 20 \mathrm{~g}, \mathrm{n}=18$; WT: $419 \pm 23 \mathrm{~g}, \mathrm{n}=26$ ). However, they appeared healthy and active with no other visible signs of illness. 
Inflammatory response to endotoxin. While serum concentrations of IL- 6 , IFN- $\gamma$, and TNF- $\alpha$ were below the limit of detection in saline treated animals, levels were induced following administration of 5 $\mathrm{mg} \mathrm{kg}{ }^{-1}$ LPS (Fig. 1). Since all samples from saline treated animals were below the detection limit we did not perform statistical analysis between saline treated and endotoxin treated. However as compared to WT, HIV-Tg animals produced significantly lower levels of IFN- $\gamma$ in response to endotoxin (Fig. 1B). Endotoxin induced IL- 6 and TNF- $\alpha$ levels to a similar extent in WT and HIV-Tg rats. While endotoxin administration significantly increased the renal mRNA expression of IL- 6 and IL-1 $\beta$ in both WT and HIV-Tg animals (Fig. 2), the renal mRNA expression of TNF- $\alpha$ and IL-10 were not affected.

Effect of endotoxin on renal transporter expression in HIV-Tg and WT. Results are summarized in Table 2.

ABC transporters. As compared to saline controls, endotoxin significantly downregulated the renal mRNA expression of Mdrla in WT and HIV-Tg rats (Fig. 3A). The expression of Mdrlb was also lower after endotoxin treatment in both WT and HIV-Tg groups, however, this did not reach significance. While endotoxin significantly downregulated the protein expression of P-gp (encoded by Mdrla and Mdr1b) in HIV-Tg animals, it only caused a trend toward a decrease in WT group $(\mathrm{P}=0.18)$ (Fig. 3B and $3 \mathrm{C}$ ). Endotoxin did not impact the mRNA expression of Mrp2 and Mrp4 in either the WT or HIV-Tg groups.

Organic cation transporters. As compared to saline controls, endotoxin significantly decreased Oct2, Oct3, Octn1 and Mate1 mRNA levels in WT but not HIV-Tg rats (Fig. 4A). Endotoxin did not impact the mRNA expression of Oct1 or Octn2 in either the WT or HIV-Tg groups. As compared to controls, the protein expression of Oct 2 and Oct 3 tended to be lower in the endotoxin treated WT animals, although this did not reach significance. As compared to saline controls, endotoxin administration did not significantly alter the protein expression of Mate 1 in either HIV or WT groups (Fig 4B and C), nevertheless, protein expression was significantly lower in the endotoxin treated HIV group as compared to endotoxin-treated WT (Fig 4B).

Organic anion transporters. As compared to controls, endotoxin caused a significant decrease in the expression of Oat2 and Urat 1 mRNA in WT but not HIV-Tg (Fig 5A). While there was a pronounced trend toward decreased expression of Oatp $4 \mathrm{c} 1$ mRNA after endotoxin administration in both WT and HIV-Tg groups, this did not reach significance $(\mathrm{P}=0.05)$. The mRNA expression of Oat 1 and Oat 3 were not significantly altered. Endotoxin administration did not significantly alter the protein expression of Oat2 and Urat1 (Fig. 5B, 5C).

\section{Kidney}

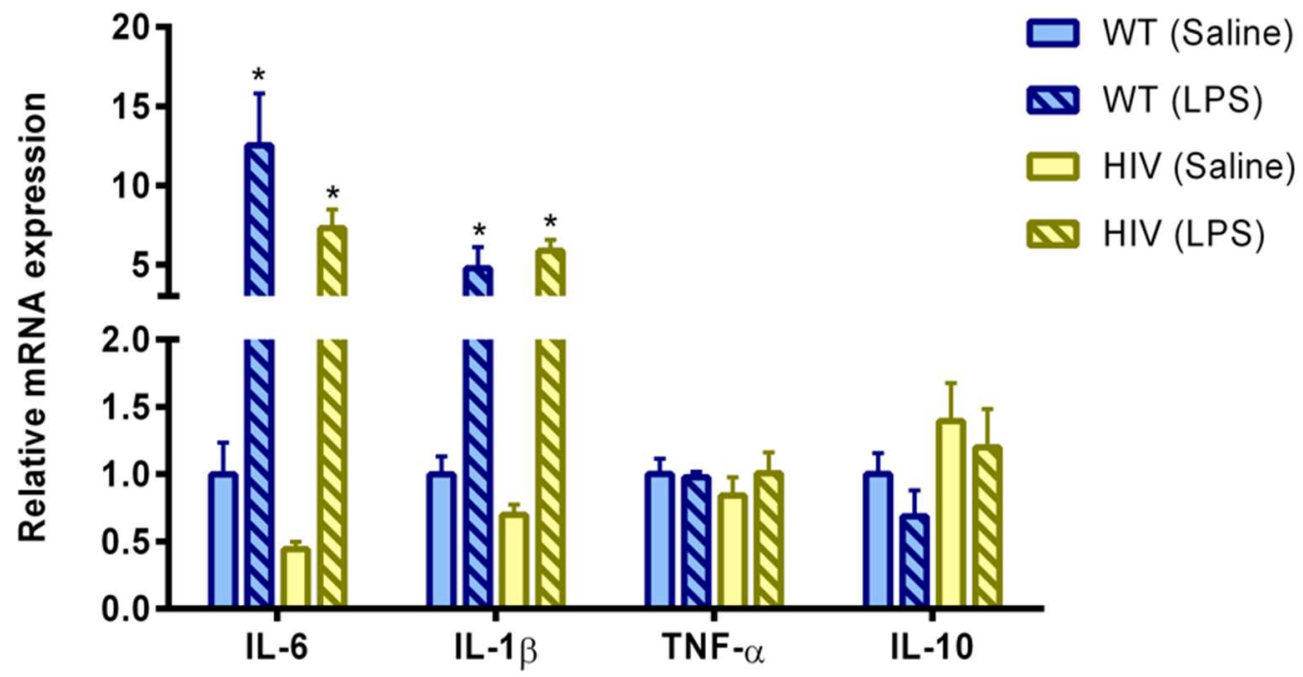

Figure 2. Effect of endotoxin on expression of cytokines in the kidneys. The relative mRNA expression of cytokines in the kidneys collected $18 \mathrm{~h}$ after i.p. administration of saline or $5 \mathrm{mg} \mathrm{kg}^{-1} \mathrm{LPS}$ to HIV-Tg and WT rats (n=7/group). mRNA levels were quantified by qRT-PCR relative to control WT and presented as mean \pm S.E.M. One-way ANOVA with Bonferroni multiple comparison test was used to determine the statistical differences among the groups. * Significantly different from saline controls $(* \mathrm{p}<0.05)$. 

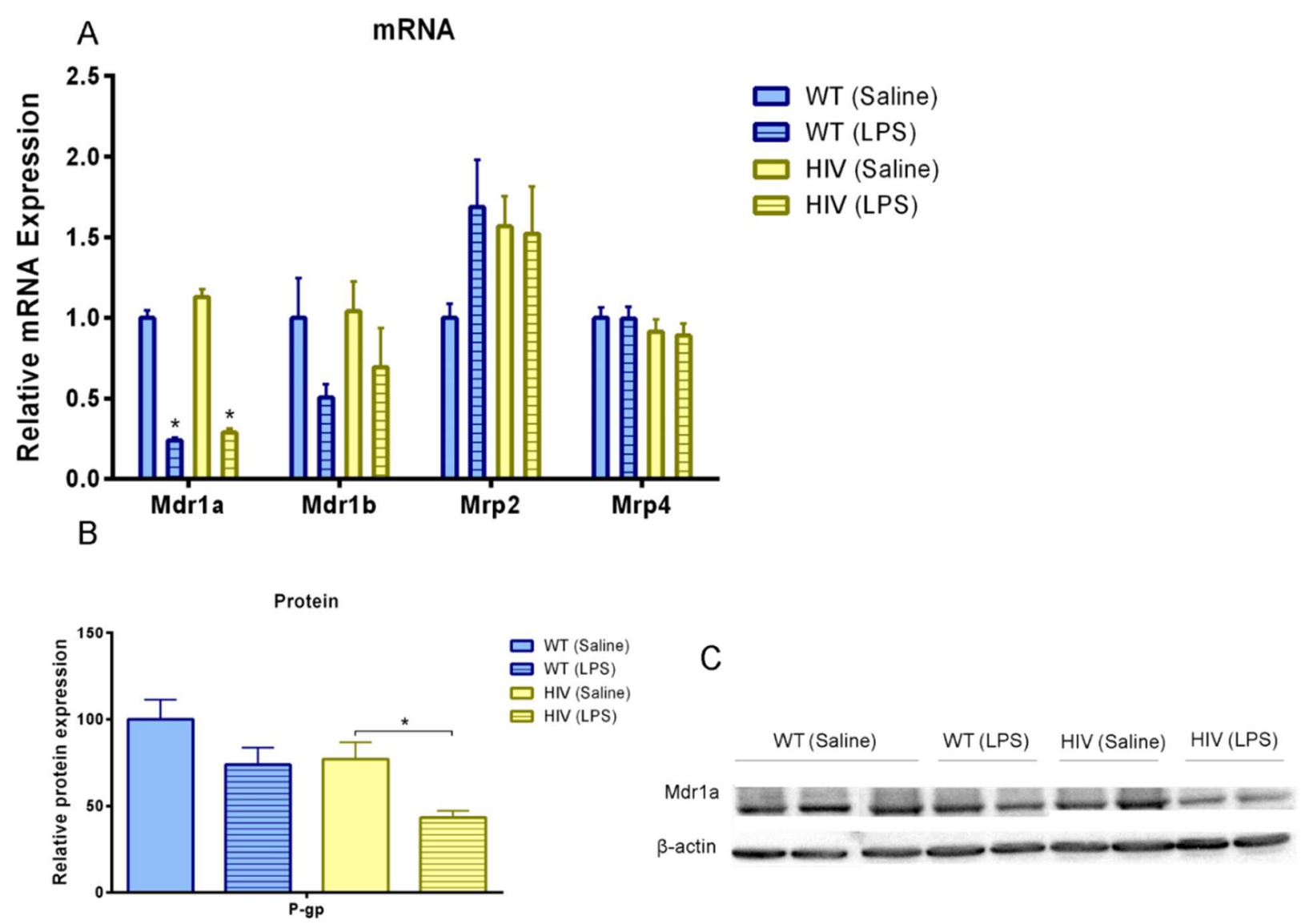

Figure 3. Renal expression of ABC transporters. (A) Relative mRNA expression of ABC transporters, (B) Protein expression of P-gp and (C) Representative western blot of P-gp. RNA and protein were extracted from kidneys collected 18 $\mathrm{h}$ after i.p. administration of saline or $5 \mathrm{mg} \mathrm{kg}^{-1}$ LPS to WT and HIV-Tg rats ( $\mathrm{n}=7 /$ group). Results are expressed relative to control WT as mean \pm S.E.M. One-way ANOVA with Bonferroni multiple comparison test was used to determine the statistical differences among the groups. * Significantly different from saline controls $(* \mathrm{p}<0.05)$.

Nucleoside and peptide transporters. As compared to saline controls, administration of endotoxin resulted in a significant downregulation of Ent1 in WT but not HIV-Tg rats while levels of Ent2 were unchanged (Fig. 6A). As compared to controls, administration of endotoxin imposed a significant increase in mRNA levels of Pept1 in both WT and HIV-Tg animals, while levels of Pept2 were significantly decreased (Fig. 6A). Consistent with mRNA data, endotoxin administration decreased protein expression of Ent1 in the WT group (Fig. 6B, 6C). Although protein expression of Pept 1 was higher in the endotoxin treated rats, this only reached significance for the HIV-Tg group (Fig. 6B, 6C).

\section{Basal expression of transporters in HIV-Tg and} WT rats. As compared to WT controls, the mRNA expressions of Oct2, Oct3, Octn1, Mate1 (Fig 4A), Urat1 (Fig 5A) and Ent1 (Fig 6A) were significantly lower in the HIV-Tg controls. In contrast, the mRNA levels of Ent2 and Pept2 (Fig 4A) were significantly higher in the HIV-Tg controls.

\section{DISCUSSION}

Dysregulation of drug transporters during inflammation has been previously reported for several tissues $(6,7)$. However, much less is known about the effects on renal transporters. HIV-infected patients are often in a state of immune activation because of exposure to bacterial endotoxin either as opportunistic infections due to immune dysregulation, or microbial translocation to the systemic circulation. Renal drug transporters play an important role in the disposition of many antiretroviral and therapeutic agents. Therefore, the possibility of altered renal drug clearance exists in HIV-infected patients. 

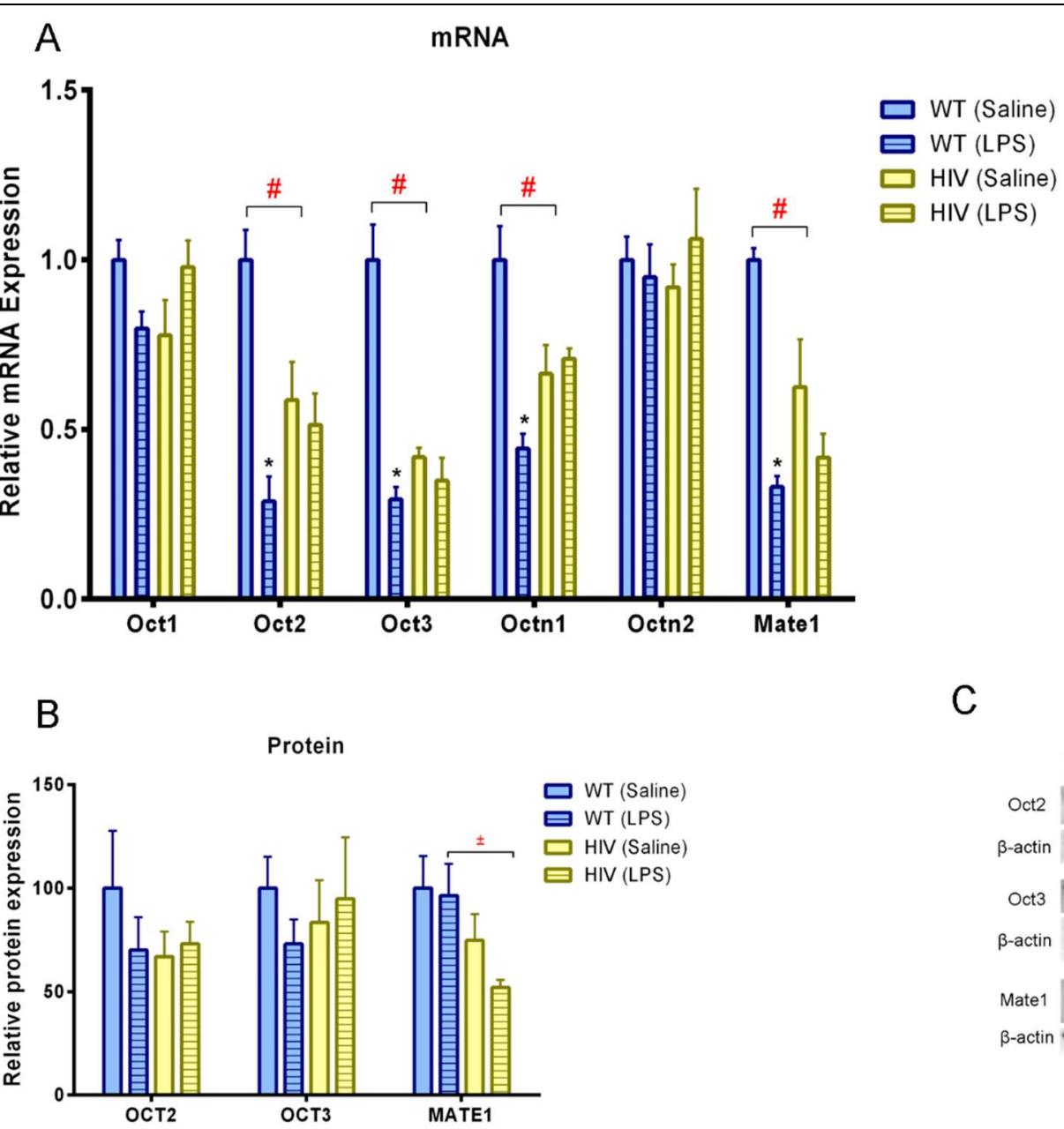

C

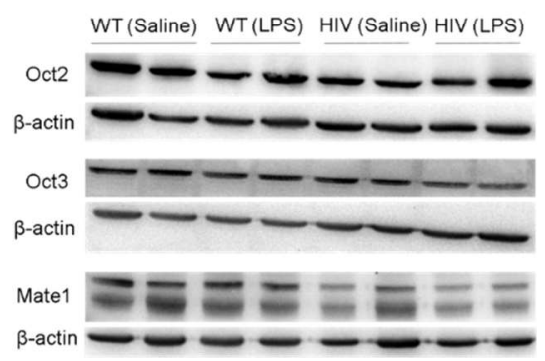

Figure 4. Renal expression of organic cation transporters. (A) Relative mRNA expression of organic cation transporters, (B) Protein expression of OCT2, OCT3 and MATE1 and (C) Representative western blots of OCT2, OCT3 and MATE1. RNA and protein were extracted from kidneys collected $18 \mathrm{~h}$ after i.p. administration of saline or $5 \mathrm{mg} \mathrm{kg}^{-1} \mathrm{LPS}_{\text {to }}$ WT and HIV-Tg rats ( $n=7 /$ group). Results are expressed relative to control WT as mean \pm S.E.M. One-way ANOVA with Bonferroni multiple comparison test was used to determine the statistical differences among the groups. * Significantly different from saline controls $(* \mathrm{p}<0.05)$; \# Significantly different from saline treated WT (\# $\mathrm{p}<0.05)$; \pm Significantly different from LPStreated WT $( \pm \mathrm{p}<0.05)$.

Preclinical and clinal studies show that HIV disease and proteins impact the expression of drug transporters, possibly due to the dysregulation of the immune system and stimulation of inflammatory cytokines. Therefore, we predicted higher levels of inflammation to occur after endotoxin exposure which would cause a more pronounced impact of endotoxemia in the HIV-Tg rats. Overall, we found administration of endotoxin induced the release of serum pro-inflammatory cytokines (IL-6, TNF- $\alpha$, IFN- $\gamma$ ) in both WT and HIV-Tg rats. This was associated with altered mRNA expression of several transporters in the kidneys. However, as compared to endotoxin treated WT, we did not observe any further endotoxin-mediated downregulation in the
HIV-Tg, contrary to what we had hypothesized. Indeed, for several transporters we saw decreased endotoxin-mediated downregulation which could stem from the lower basal expression observed in the HIV-Tg group.

Endotoxin induced the release of proinflammatory cytokines in both WT and HIV-Tg rats. However, a significantly lower induction of IFN- $\gamma$ was seen in the HIV-Tg animals. This agrees with previous studies which have reported that HIV patients have a decreased production of IFN- $\gamma \quad(24$, 25). It is also in line with Reid W., et al., who demonstrated that peripheral blood mononuclear cells (PBMCs) extracted from HIV-Tg rats produced 


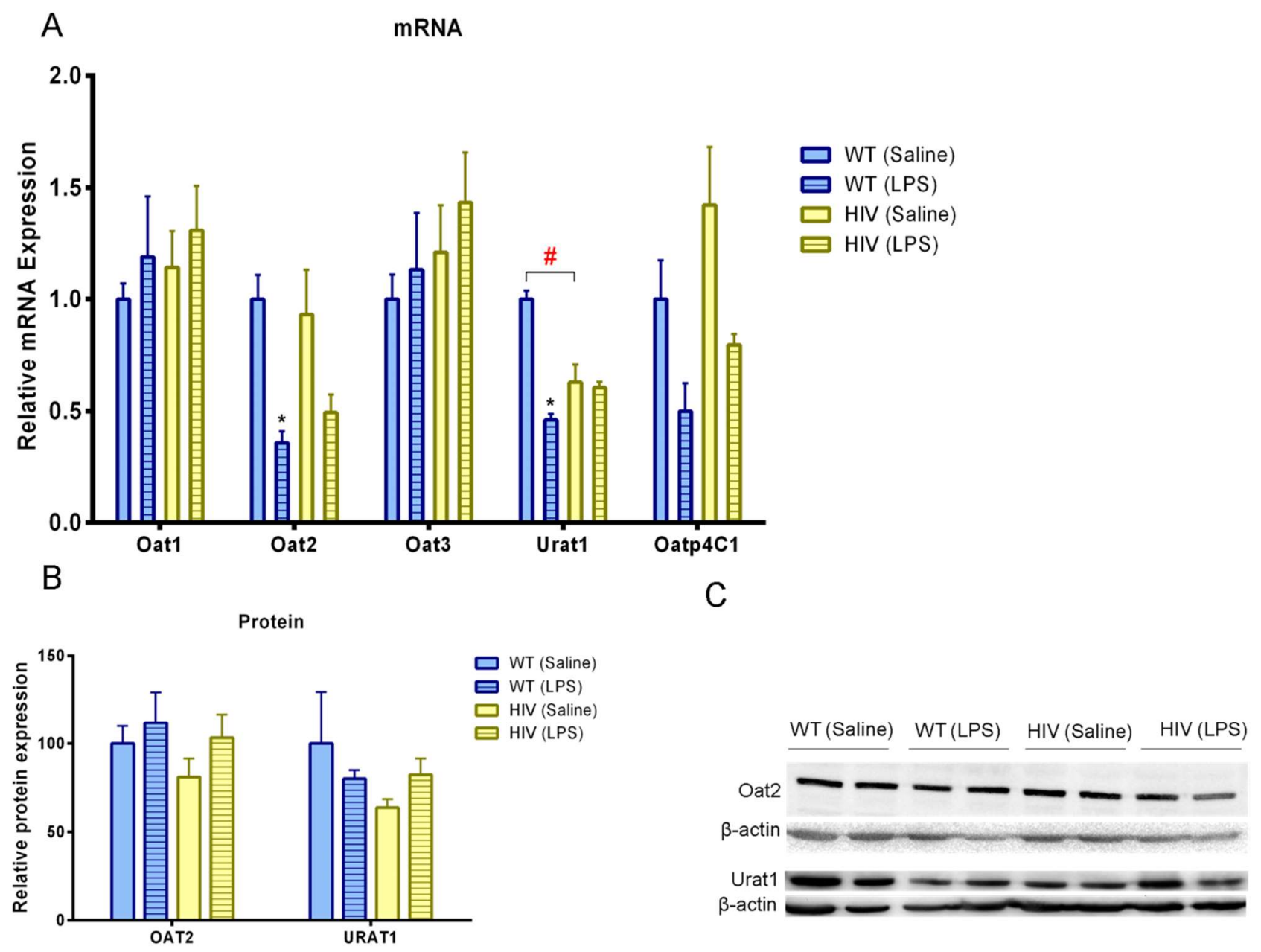

Figure 5. Renal expression of organic anion transporters. (A) Relative mRNA expression of organic anion transporters, (B) Protein expression of OAT2 and URAT1 and (C) Representative western blots of OAT2 and URAT1. RNA and protein were extracted from kidneys collected $18 \mathrm{~h}$ after i.p. administration of saline or $5 \mathrm{mg} \mathrm{kg}^{-1}$ LPS to WT and HIV-Tg rats $(\mathrm{n}=7 /$ group). Results are expressed relative to control WT as mean \pm S.E.M. One-way ANOVA with Bonferroni multiple comparison test was used to determine the statistical differences among the groups. * Significantly different from saline controls $(* \mathrm{p}<0.05)$; \# Significantly different from WT (\# $\mathrm{p}<0.05)$.

significantly less IFN- $\gamma$ compared to WT following in vitro stimulation by phorbol 12-myristrate 13acetate and ionomycin (26). This has been attributed to the reduction of $\mathrm{T}$ lymphocytes and increased apoptosis $(19,26,27)$. On the other hand, we observed a similar induction of IL- 6 and TNF- $\alpha$ in HIV-Tg and WT rats after endotoxin administration. In HIV-Tg rats, it has been reported that loss of $\mathrm{T}$ lymphocytes causes immune dysfunction via dysregulation of Th1 effector cells (27). Th1 cells are mainly involved in cell mediated immunity, producing IFN- $\gamma$, IL-2 and TNF- $\alpha$, while Th 2 cells which are functional in the transgenic animals, play an important role in humoral immunity and IL-4, IL-
5, IL-6, and IL-10 production (27). Thus, it appears that while interferon levels may be somewhat lower in the HIV-Tg animals, other inflammatory responses are intact.

We found that endotoxin administration resulted in a significant reduction in both mRNA and protein expression of Mdrla/P-gp which is an important efflux drug transporter located on the apical side of the tubules (28). P-gp is involved in the efflux of endogenous compounds such as steroids, lipids, bilirubin and bile acids and plays an important role in the disposition and distribution of the commonly prescribed antiviral agents ritonavir and zidovudine. 

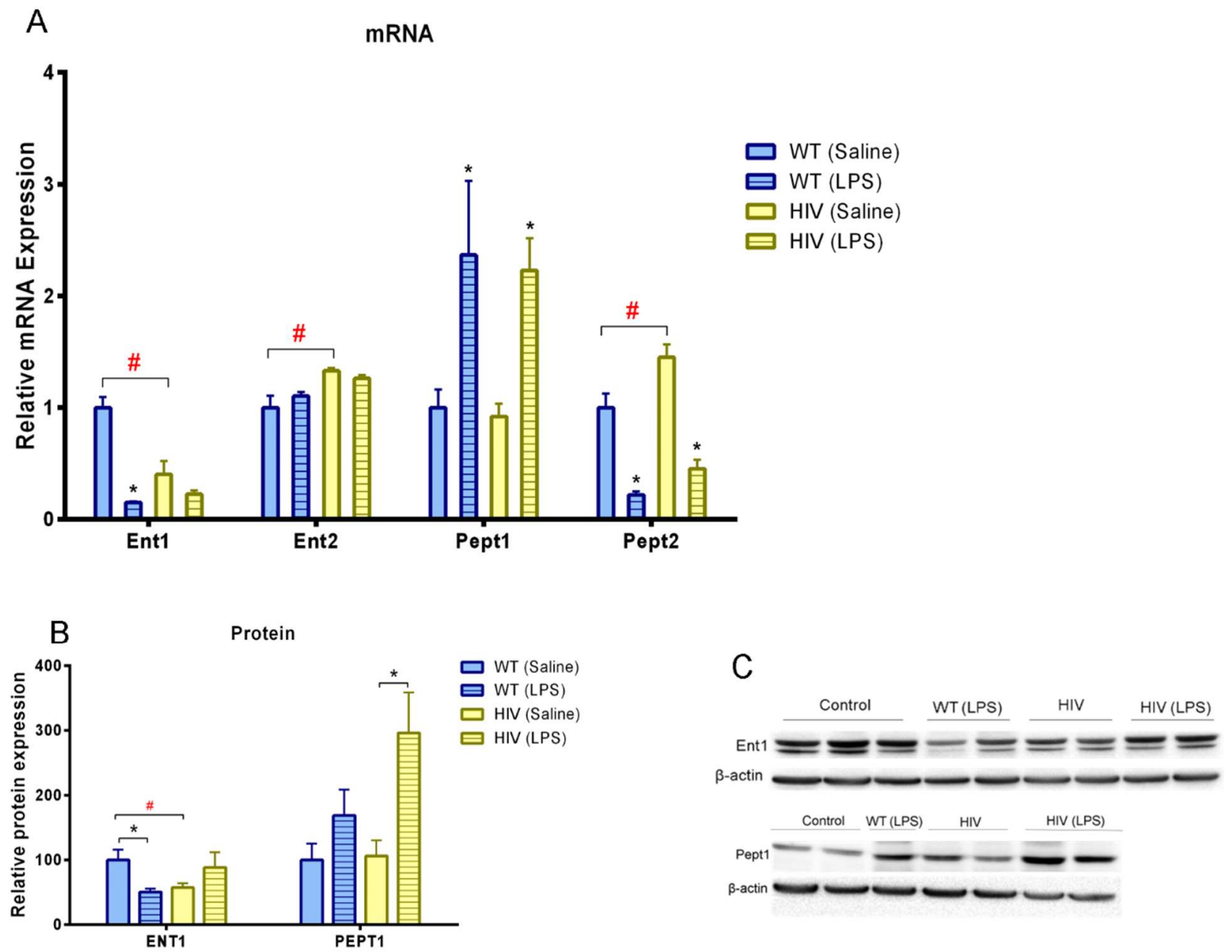

Figure 6. Renal expression of nucleoside and peptide transporters. (A) Relative mRNA expression of nucleoside and peptide transporters, (B) Protein expression of ENT1 and PEPT1 and (C) Representative western blots of ENT1 and PEPT1. RNA and protein were extracted from kidneys collected $18 \mathrm{~h}$ after i.p. administration of saline or $5 \mathrm{mg} \mathrm{kg}^{-1} \mathrm{LPS}$ to WT and HIV-Tg rats ( $\mathrm{n}=7$ /group). Results are expressed relative to control WT as mean \pm S.E.M. One-way ANOVA with Bonferroni multiple comparison test was used to determine the statistical differences among the groups. * Significantly different from saline controls $(* \mathrm{p}<0.05)$; \# Significantly different from saline treated WT $(\# \mathrm{p}<0.05)$.

Our results are consistent with those of Ando H., et al. (2001) who demonstrated an endotoxin mediated downregulation of Mdr1a expression and activity in the kidney of rats (29). This is also in line with previous studies which have shown an inflammation-mediated downregulation of Mdr1a mRNA and protein in the liver, brain and intestine (7). It is possible that downregulation of P-gp could reduce the renal excretion of drug substrates resulting in increased accumulation and toxicity.

Our study demonstrated that endotoxin mediates changes in the mRNA expression of several organic cation transporters within the kidney. Endotoxin imposed a significant downregulation of Oct2, Oct3,
Octn1 and Mate1 in WT but not HIV-Tg, however the basal expression of these transporters was already significantly lower in the HIV-Tg controls. Therefore, differences in the response to endotoxin could be due to the limited expression of these transporters in the HIV-Tg rats. Moreover, the lower basal expression of these transporters may stem from changes due to HIV viral proteins. While decreased renal mRNA expression of Oct2 was previously reported in endotoxin treated rats (30), the impact on Oct3, Octn1 and Mate1 has not been examined. OCT2 and MATE1 are among the main organic cation transporters in the kidney that are located on the basolateral and apical sides of the proximal 
tubules, respectively. OCT2 mediates the uptake of organic cations from the blood to the proximal tubular cells for secretion (31) and plays an important role in renal clearance of endogenous compounds such as monoamine neurotransmitters (32), creatinine (33) and drugs such as metformin and cimetidine (34). Located on the luminal side, MATE1 is involved in the renal secretion of creatinine (35) and therapeutic drugs such as metformin (36) and cephalexin (37). Also expressed on the luminal side, OCTN1 is involved in the renal reabsorption of L-carnitine (38) and transport of several chemotherapeutics such as doxorubicin and methotrexate (39). As these organic cation transporters play an important role in the renal clearance of drug substrates, downregulation in their expression could have clinical consequences such as altered systemic drug concentrations and nephrotoxicity. However, despite alterations at the mRNA level, we did not observe a significant change in the protein expression of these transporters. The stability or half-life of these membrane transporters has not been fully elucidated hence it is possible that despite the reduction in transcripts, a period of 18 hours may not have been sufficient to detect substantial changes in protein expression or activity. Studies at later time points are clearly needed to determine the potential clinical consequences of these endotoxin mediated changes.

Our results demonstrated that endotoxin mediates changes in the renal mRNA expression of organic anion transporters in the kidney. In WT rats, endotoxin significantly reduced the mRNA levels of Urat1, a transporter that is involved in reabsorption and regulation of uric acid (40). However, as discussed above, no significant effect was seen at the protein level. We also saw a significant decrease in the expression of Oat2 in the WT group. The OAT transporters are involved in the transport of prostaglandins, uric acid (41), pravastatin (42), and most nonsteroidal anti-inflammatory drugs (43). However, as OAT1 and OAT3 are the most abundantly expressed organic anion transporters in the kidney [42], and mRNA levels of these transporters were unchanged, the clinical significance of our findings is unclear. In contrast, Hocherl et al. reported a dose and time dependent reduction in the mRNA expression of Oat1 and Oat3 in rat kidney after i.v. administration of LPS (44). Increased inflammation or disease severity after intravenous endotoxin doses likely account for dissimilar findings.
Endotoxin imposed a significant downregulation in the expression of the Ent1 in WT but not HIV-Tg. As basal expression of Ent 1 was dramatically lower in the HIV-Tg rats, it is likely that endotoxin could not further impact its expression. It is likely that changes in basal expression stem from HIV viral protein induced changes. Although ENT1 mediates the transport of anticancer and antiviral nucleoside analogs (45), it is a bidirectional transporter, therefore the clinical consequence of downregulation is not clear. While we observed an endotoxin mediated upregulation of Pept1 mRNA and protein expression, the mRNA expression of Pept2 was significantly decreased. As both PEPT2 and PEPT1 are involved in the reabsorption of di- and tripeptides and peptidomimetic substrates in the kidneys (46), upregulation of Pept 2 could be a homeostatic response to Pept1 downregulation.

As compared to WT, HIV-Tg rats demonstrated significant differences in the basal expression of several SLC uptake transporters while no differences were detected with the ABC efflux transporters. Previously, Robillard et al reported an increase in the basal mRNA levels of several ABC transporters in HIV-Tg Sprague Dawley rats as compared to nonlittermate WT (15). This discrepancy could be due to differences in strain or control animals. Dysregulation of transporter expression is likely due to viral protein-mediated activation of inflammatory responses. Indeed, it was previously reported that treatment of cultured murine and human astrocytes with the HIV protein gp120 stimulated release of proinflammatory cytokines, resulting in downregulation of P-gp $(13,14)$. These studies support the potential role of HIV proteins in the dysregulation of transporters. Previous studies have demonstrated increased chronic oxidative stress in HIV-infected patients (47). Therefore, changes in transporter expression could also occur through oxidative stress-mediated pathways. Oxidative stress is known to activate nuclear factor kappa-lightchain-enhancer of activated $B$ cells (NF-kB), a transcription factor which has recently been implicated in endotoxin mediated downregulation of hepatic transporters in mice (48).

Our study provides in vivo evidence that HIV and endotoxin modulate the expression of several drug transporters in the kidney. Changes in the expression of renal transporters could result in alterations in the excretion and disposition of endogenous and exogenous substrates of these transporters. At this point the clinical significance of 
endotoxin mediated alterations of the renal drug transporters is not clear as endotoxin mediated effects were only examined at 18 hours and protein levels did not always correspond to mRNA changes at this time point. Further studies are needed to determine whether infection or inflammationmediated alterations impact the renal clearance and disposition of clinically important drug substrates.

\section{ACKNOWLEDGMENT}

The authors thank Yen Ting Shen for his technical assistance. Funding for this study was provided by an operating grant from the Canadian Institutes of Health Research [MOP 13346].

Table 2. Summary of endotoxin-mediated changes in WT and HIV-Tg rats. Data is presented as the \% change in expression in endotoxin treated rats as compared to their saline controls. \# Not significantly changed from saline controls. Student's t-test was used to analyze data between HIV-Tg and WT.* $\mathrm{P}<0.05$, ** $\mathrm{P}<0.01$, *** $\mathrm{P}<0.001 \mathrm{NS}$ : \% change not significantly different between WT and HIV-Tg.

\begin{tabular}{|c|c|c|c|}
\hline Drug Transporter & $\%$ Change in WT & \% Change in HIV-Tg & HIV-Tg vs WT \\
\hline \multicolumn{4}{|l|}{ mRNA } \\
\hline Mdr1a & $-76 \pm 5$ & $-74 \pm 5$ & NS \\
\hline Mdr1b & $\#$ & $\#$ & --- \\
\hline Mrp2 & $\#$ & \# & --- \\
\hline Mrp4 & \# & \# & --- \\
\hline Oct1 & $\#$ & $\#$ & --- \\
\hline Oct2 & $-71 \pm 18$ & $-12 \pm 38(\#)$ & $* *$ \\
\hline Oct3 & $-71 \pm 8$ & $-17 \pm 43(\#)$ & $*$ \\
\hline Octn1 & $-56 \pm 10$ & $7 \pm 11(\#)$ & $* * *$ \\
\hline Octn2 & $\#$ & $\#$ & --- \\
\hline Mate1 & $-67 \pm 8$ & $33 \pm 27(\#)$ & $*$ \\
\hline Oat1 & \# & \# & --- \\
\hline Oat2 & $-64 \pm 12$ & $-47 \pm 23(\#)$ & NS \\
\hline Oat3 & $\#$ & $\#$ & --- \\
\hline Urat1 & $-54 \pm 6$ & $-4 \pm 10(\#)$ & $* * *$ \\
\hline Oatp4c1 & \# & \# & --- \\
\hline Ent1 & $-85 \pm 2$ & $-44 \pm 21(\#)$ & $* *$ \\
\hline Ent2 & \# & \# & --- \\
\hline Pept1 & $137 \pm 163$ & $145 \pm 84$ & NS \\
\hline Pept2 & $-78 \pm 8$ & $-69 \pm 14$ & NS \\
\hline IL-6 & $1150 \pm 865$ & $1550 \pm 651$ & NS \\
\hline IL-1 $\beta$ & $377 \pm 350$ & $742 \pm 256$ & $*$ \\
\hline TNF- $\alpha$ & \# & \# & --- \\
\hline IL-10 & \# & \# & --- \\
\hline \multicolumn{4}{|l|}{ Proteins } \\
\hline Ent1 & $-50 \pm 11$ & $54 \pm 100(\#)$ & $*$ \\
\hline Mate1 & \# & \# & --- \\
\hline Oat2 & \# & \# & --- \\
\hline Oct 2 & $\#$ & $\#$ & --- \\
\hline Oct3 & \# & \# & --- \\
\hline Pept1 & $68 \pm 98(\#)$ & $179 \pm 131$ & NS \\
\hline P-gp & $-26 \pm 20(\#)$ & $-43 \pm 10(\#)$ & NS \\
\hline Urat1 & \# & $\#$ & --- \\
\hline
\end{tabular}

\section{REFERENCES}

1. Hellerstein M, Hanley MB, Cesar D, Siler S, Papageorgopoulos C, Wieder E, et al. Directly measured kinetics of circulating $\mathrm{T}$ lymphocytes in normal and HIV-1-infected humans. Nat Med. 1999;5(1):83-9.

2. Hazenberg MD, Stuart JW, Otto SA, Borleffs JC, Boucher CA, de Boer RJ, et al. T-cell division in human immunodeficiency virus (HIV)-1 infection is mainly due to immune activation: a longitudinal 
analysis in patients before and during highly active antiretroviral therapy (HAART). Blood. 2000;95(1):249-55.

3. Brenchley JM, Price DA, Schacker TW, Asher TE, Silvestri G, Rao S, et al. Microbial translocation is a cause of systemic immune activation in chronic HIV infection. Nat Med. 2006;12(12):1365-71.

4. Nazli A, Chan O, Dobson-Belaire WN, Ouellet M, Tremblay MJ, Gray-Owen SD, et al. Exposure to HIV-1 directly impairs mucosal epithelial barrier integrity allowing microbial translocation. PLoS Pathog. 2010;6(4):e1000852.

5. Lu YC, Yeh WC, Ohashi PS. LPS/TLR4 signal transduction pathway. Cytokine. 2008;42(2):145-51.

6. Petrovic V, Teng S, Piquette-Miller M. Regulation of drug transporters during infection and inflammation. Mol Interv. 2007;7(2):99-111.

7. Morgan ET, Goralski KB, Piquette-Miller M, Renton KW, Robertson GR, Chaluvadi MR, et al. Regulation of drug-metabolizing enzymes and transporters in infection, inflammation, and cancer. Drug Metab Dispos. 2008;36(2):205-16.

8. Teng S, Piquette-Miller M. Regulation of transporters by nuclear hormone receptors: implications during inflammation. Mol Pharm. 2008;5(1):67-76.

9. Shedlofsky SI, Israel BC, McClain CJ, Hill DB, Blouin RA. Endotoxin administration to humans inhibits hepatic cytochrome P450-mediated drug metabolism. J Clin Invest. 1994;94(6):2209-14.

10. Nigam SK. What do drug transporters really do? Nat Rev Drug Discov. 2015;14(1):29-44.

11. Hillgren KM, Keppler D, Zur AA, Giacomini KM, Stieger B, Cass CE, et al. Emerging transporters of clinical importance: an update from the International Transporter Consortium. Clin Pharmacol Ther. 2013;94(1):52-63.

12. Hayashi K, Pu H, Andras IE, Eum SY, Yamauchi A, Hennig B, et al. HIV-TAT protein upregulates expression of multidrug resistance protein 1 in the blood-brain barrier. J Cereb Blood Flow Metab. 2006;26(8):1052-65.

13. Ashraf T, Ronaldson PT, Persidsky Y, Bendayan R. Regulation of P-glycoprotein by human immunodeficiency virus-1 in primary cultures of human fetal astrocytes. J Neurosci Res. 2011;89(11):1773-82.

14. Ronaldson PT, Bendayan R. HIV-1 viral envelope glycoprotein gp120 triggers an inflammatory response in cultured rat astrocytes and regulates the functional expression of P-glycoprotein. Mol Pharmacol. 2006;70(3):1087-98.

15. Robillard KR, Hoque MT, Bendayan R. Expression of ATP-binding cassette membrane transporters in a HIV-1 transgenic rat model. Biochem Biophys Res Commun. 2014;444(4):531-6.

16. Kis O, Sankaran-Walters S, Hoque MT, Walmsley SL, Dandekar S, Bendayan R. HIV-1 Alters Intestinal
Expression of Drug Transporters and Metabolic Enzymes: Implications for Antiretroviral Drug Disposition. Antimicrob Agents Chemother. 2016;60(5):2771-81.

17. De Rosa MF, Robillard KR, Kim CJ, Hoque MT, Kandel G, Kovacs C, et al. Expression of membrane drug efflux transporters in the sigmoid colon of HIVinfected and uninfected men. J Clin Pharmacol. 2013;53(9):934-45.

18. Ivanyuk A, Livio F, Biollaz J, Buclin T. Renal Drug Transporters and Drug Interactions. Clin Pharmacokinet. 2017;56(8):825-92.

19. Reid W, Sadowska M, Denaro F, Rao S, Foulke J, Jr., Hayes N, et al. An HIV-1 transgenic rat that develops HIV-related pathology and immunologic dysfunction. Proc Natl Acad Sci U S A. 2001;98(16):9271-6.

20. Peng J, Vigorito M, Liu X, Zhou D, Wu X, Chang SL. The HIV-1 transgenic rat as a model for HIV-1 infected individuals on HAART. J Neuroimmunol. 2010;218(1-2):94-101.

21. Abbondanzo SJ, Chang SL. HIV-1 transgenic rats display alterations in immunophenotype and cellular responses associated with aging. PLoS One. 2014;9(8):e105256.

22. Ghoneim RH, Piquette-Miller M. EndotoxinMediated Downregulation of Hepatic Drug Transporters in HIV-1 Transgenic Rats. Drug Metab Dispos. 2016;44(5):709-19.

23. Petrovic V, Piquette-Miller M. Impact of polyinosinic/polycytidylic acid on placental and hepatobiliary drug transporters in pregnant rats. Drug Metab Dispos. 2010;38(10):1760-6.

24. Bailer RT, Holloway A, Sun J, Margolick JB, Martin M, Kostman J, et al. IL-13 and IFN-gamma secretion by activated $\mathrm{T}$ cells in HIV-1 infection associated with viral suppression and a lack of disease progression. J Immunol. 1999;162(12):7534-42.

25. Ostrowski SR, Gerstoft J, Pedersen BK, Ullum H. Impaired production of cytokines is an independent predictor of mortality in HIV-1-infected patients. AIDS. 2003;17(4):521-30.

26. Reid W, Abdelwahab S, Sadowska M, Huso D, Neal A, Ahearn A, et al. HIV-1 transgenic rats develop T cell abnormalities. Virology. 2004;321(1):111-9.

27. Yadav A, Pati S, Nyugen A, Barabitskaja O, Mondal $\mathrm{P}$, Anderson M, et al. HIV-1 transgenic rat CD4+ T cells develop decreased CD28 responsiveness and suboptimal Lck tyrosine dephosphorylation following activation. Virology. 2006;353(2):357-65.

28. Sharom FJ. The P-glycoprotein multidrug transporter. Essays Biochem. 2011;50(1):161-78.

29. Ando H, Nishio Y, Ito K, Nakao A, Wang L, Zhao YL, et al. Effect of endotoxin on P-glycoproteinmediated biliary and renal excretion of rhodamine123 in rats. Antimicrob Agents Chemother. 2001;45(12):3462-7. 
30. Heemskerk S, Wouterse AC, Russel FG, Masereeuw $R$. Nitric oxide down-regulates the expression of organic cation transporters (OCT) 1 and 2 in rat kidney during endotoxemia. Eur J Pharmacol. 2008;584(2-3):390-7.

31. Koepsell H, Lips K, Volk C. Polyspecific organic cation transporters: structure, function, physiological roles, and biopharmaceutical implications. Pharm Res. 2007;24(7):1227-51.

32. Busch AE, Karbach U, Miska D, Gorboulev V, Akhoundova A, Volk C, et al. Human neurons express the polyspecific cation transporter hOCT2, which translocates monoamine neurotransmitters, amantadine, and memantine. Mol Pharmacol. 1998;54(2):342-52.

33. Urakami Y, Kimura N, Okuda M, Inui K. Creatinine transport by basolateral organic cation transporter hOCT2 in the human kidney. Pharm Res. 2004;21(6):976-81.

34. Wang ZJ, Yin OQ, Tomlinson B, Chow MS. OCT2 polymorphisms and in-vivo renal functional consequence: studies with metformin and cimetidine. Pharmacogenet Genomics. 2008;18(7):637-45.

35. Tanihara Y, Masuda S, Sato T, Katsura T, Ogawa O, Inui K. Substrate specificity of MATE1 and MATE2$\mathrm{K}$, human multidrug and toxin extrusions $/ \mathrm{H}(+)-$ organic cation antiporters. Biochem Pharmacol. 2007;74(2):359-71.

36. Tsuda M, Terada T, Ueba M, Sato T, Masuda S, Katsura $\mathrm{T}$, et al. Involvement of human multidrug and toxin extrusion 1 in the drug interaction between cimetidine and metformin in renal epithelial cells. $\mathrm{J}$ Pharmacol Exp Ther. 2009;329(1):185-91.

37. Watanabe $S$, Tsuda M, Terada $T$, Katsura $T$, Inui K. Reduced renal clearance of a zwitterionic substrate cephalexin in MATE1-deficient mice. J Pharmacol Exp Ther. 2010;334(2):651-6.

38. Garrett Q, Xu S, Simmons PA, Vehige J, Flanagan $\mathrm{JL}$, Willcox MD. Expression and localization of carnitine/organic cation transporter OCTN1 and OCTN2 in ocular epithelium. Invest Ophthalmol Vis Sci. 2008;49(11):4844-9.
39. Okabe M, Szakacs G, Reimers MA, Suzuki T, Hall MD, Abe T, et al. Profiling SLCO and SLC22 genes in the NCI-60 cancer cell lines to identify drug uptake transporters. Mol Cancer Ther. 2008;7(9):3081-91.

40. Enomoto A, Kimura H, Chairoungdua A, Shigeta Y, Jutabha P, Cha SH, et al. Molecular identification of a renal urate anion exchanger that regulates blood urate levels. Nature. 2002;417(6887):447-52.

41. Koepsell H, Endou H. The SLC22 drug transporter family. Pflugers Arch. 2004;447(5):666-76.

42. Khamdang S, Takeda M, Shimoda M, Noshiro R, Narikawa S, Huang XL, et al. Interactions of humanand rat-organic anion transporters with pravastatin and cimetidine. J Pharmacol Sci. 2004;94(2):197202.

43. Maeda A, Tsuruoka S, Kanai Y, Endou H, Saito K, Miyamoto E, et al. Evaluation of the interaction between nonsteroidal anti-inflammatory drugs and methotrexate using human organic anion transporter 3-transfected cells. Eur J Pharmacol. 2008;596(13):166-72.

44. Hocherl K, Schmidt C, Bucher M. COX-2 inhibition attenuates endotoxin-induced downregulation of organic anion transporters in the rat renal cortex. Kidney Int. 2009;75(4):373-80.

45. Boswell-Casteel RC, Hays FA. Equilibrative nucleoside transporters-A review. Nucleosides Nucleotides Nucleic Acids. 2017;36(1):7-30.

46. Brandsch M. Transport of drugs by proton-coupled peptide transporters: pearls and pitfalls. Expert Opin Drug Metab Toxicol. 2009;5(8):887-905.

47. Baruchel S, Wainberg MA. The role of oxidative stress in disease progression in individuals infected by the human immunodeficiency virus. J Leukoc Biol. 1992;52(1):111-4.

48. Abualsunun WA, Piquette-Miller M. Involvement of Nuclear Factor kappaB, not Pregnane X Receptor, in Inflammation-Mediated Regulation of Hepatic Transporters. Drug Metab Dispos. 2017;45(10):1077-83. 